\title{
A Predictive Model for Additions to $N$-Alkyl Pyridiniums
}

\author{
Brian J. Knight, Zachary A. Tolchin, and Joel M. Smith* \\ Department of Chemistry and Biochemistry, Florida State University, 95 Chieftan Way, Tallahassee, FL 32306
}

\begin{abstract}
Disclosed in this communication is a thorough study on the dearomative addition of organomagnesium nucleophiles to $N$-alkyl pyridinium electrophiles. The regiochemical outcomes have observable and predictable trends associated with the substituent patterns on the pyridinium electrophile. Often, the substituent effects can be either additive, giving high selectivities, or ablative, giving competing outcomes. Additionally, the nature of the organometallic nucleophilic component was also investigated for its role in the regioselective outcome. The effects of either reactive component are important to both the overall reactivity and site of nucleophilic addition. The utility of these observed trends is demonstrated in a concise, dearomative synthesis of a tricyclic compound shown to have insecticidal activity.
\end{abstract}

Heterocyclic compounds containing nitrogen atoms are ubiquitous in the realms of natural product isolation, materials chemistry, and drug discovery. ${ }^{1-5}$ In particular, as $59 \%$ of all marketed small molecule drugs contain a nitrogen heterocycle, ${ }^{6}$ direct and concise synthetic access to diverse libraries of these important moieties remains an outstanding challenge. ${ }^{7}$ Furthermore, being that the most common nitrogen heterocycles in marketed drugs are piperidine and pyridine (ranked first and second, respectively), easily functionalizing and modifying their structures is of the utmost importance to expediting therapeutic campaigns. ${ }^{8-10}$

Shown in Figure 1A are a selection of medicinally important natural products and rationally designed therapeutic agents that all contain variously substituted piperidines or tetrahydropyridines. While manzamine A $(\mathbf{1})^{11}$ and morphine $(\mathbf{2})^{12}$ contain stereochemically dense patterns within and contiguous to their piperidine rings, compounds like $3^{13}$ and Paroxetine (4), ${ }^{14}$ while arguably less complex, still pose several synthetic questions regarding their construction. The control of relative and absolute stereochemistry is a consideration for all of these targets (1-4). Furthermore, the regiochemical control of desired unsaturation (e.g. 3) poses an issue ${ }^{15}$ especially when the final product has sensitive functionality. ${ }^{16}$

Many strategies exist for the construction of piperidines, some of which are highlighted in Figure 1B. ${ }^{17,18}$ Certainly, utilizing nitrogen's innate nucleophilicity dominates most disconnection strategies where substitution or reductive amination tactics are common. Additionally, hydroamination tactics that encourage N-C2 disconnections are also employed, but these approaches are often beset by arduous construction and prefunctionalization of linear precursors. ${ }^{19}$ The reduction of lactams and imides in higher oxidation levels also remains a popular avenue for achieving the desired saturation, but controlled reduction of these carbonyls can be challenging in a complex setting, lending need for protecting groups or circuitous redox adjustments. $^{20,21}$ Thus, dearomative functionalization arose as an attractive tactic for the conversion of parent pyridine heterocycles to their reduced piperidine analogs. ${ }^{22-26}$ This type of disconnection is not without its challenges, however, as derivatization of

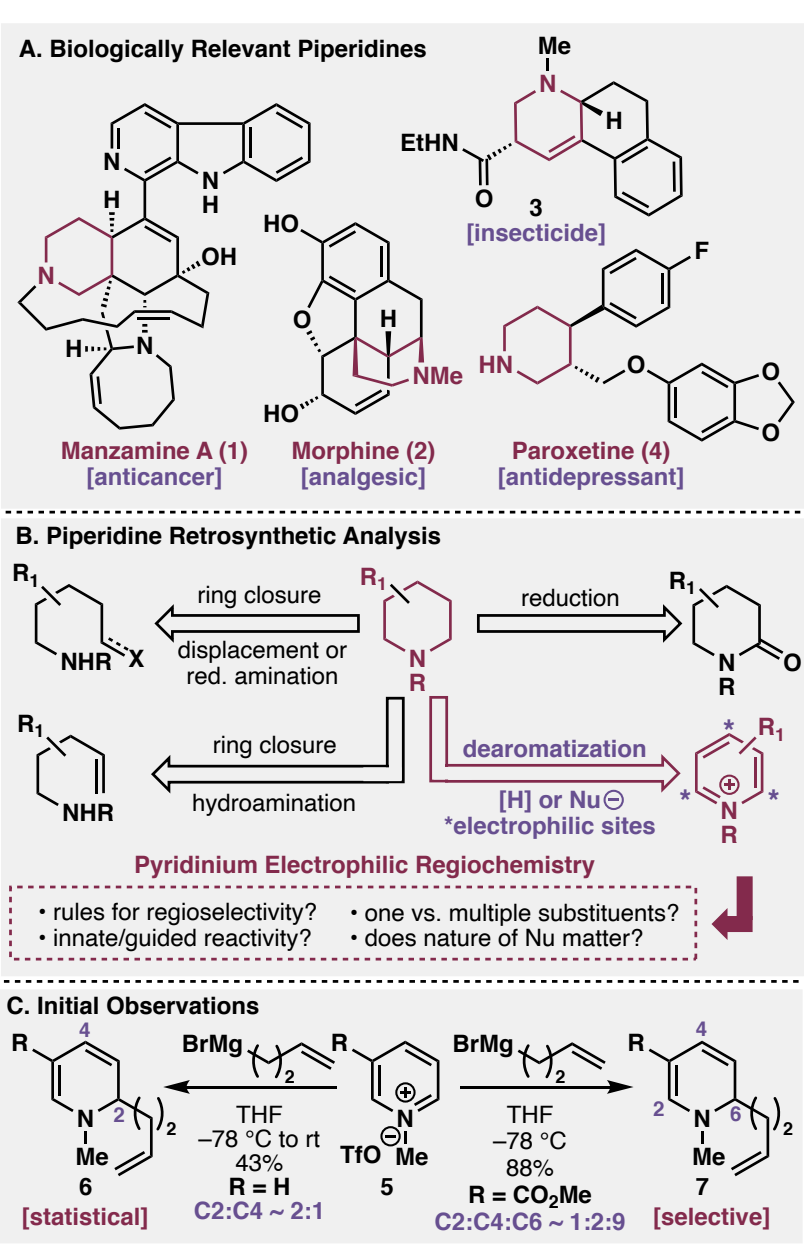

Figure 1. (A) Representative and important piperidines and tetrahydropyridines. (B) Common strategies for assembling piperidines. (C) Substituent biasing of innate regiochemical preference of pyridiniums. Yields were determined by ${ }^{1} \mathrm{H}$ NMR with an internal standard.

pyridines requires initial activation to a pyridinium ${ }^{27}$ followed by a predictable nucleophilic addition. Prior to this study, organometallic additions to some monosubstituted pyridiniums have been established, but the innate selectivity of addition to 
multiply-substituted substrates has not been well understood. ${ }^{28}$ Notable contributions made by Comins, ${ }^{29-31}$ Yamaguchi, ${ }^{32,33}$ and Donohoe $^{34}$ have laid some basic groundwork in this area. Nevertheless, understanding the innate electrophilic regioselectivity of substituted pyridiniums stands as a significant problem towards practitioners harnessing dearomative strategies. In considering factors such as steric, electronic, and chelation effects, this study aims to identify rules and guidelines understanding the regiochemical outcomes of additions to $N$-alkyl pyridiniums, enabling practitioners to more accurately predict the site of nucleophilic addition to these valuable heterocyclic synthons.

Activation of pyridines via acylation or alkylation of the nitrogen has been a realm of research for several decades. ${ }^{15}$ The study of acyl pyridinium reactivity and regioselectivity has dwarfed the studies of their $N$-alkyl counterparts, largely due to product stability and/or enhanced reactivity. Many have invoked the acyl/iminoyl group as a non-innocent activating group capable of guiding ${ }^{35-37}$ and/or sterically controlling ${ }^{38-40}$ reactivity upon the event of dearomatization. In these examples, the innate reactivity of the pyridinium intermediate is perturbed, giving a more engineered and programmatic outcome. Conversely, with an interest in pursuing targets such as $\mathbf{1 - 4}$, exploiting and controlling alkylated pyridiniums would provide a more direct and ideal route for their preparation. Initially, the activation of unsubstituted pyridine and methyl nicotinate, via activation with methyl triflate, was investigated (for ease and solubility purposes) followed by subsequent treatment with 3-butenylmagnesium bromide. By crude ${ }^{1} \mathrm{H}$ NMR, Grignard addition to the methylated pyridinium gave a near statistical mixture of possible products with a $\mathrm{C} 2$ : $\mathrm{C} 4$ ratio of roughly $2: 1$. By contrast, executing the same reaction with methyl nicotinate gave the $\mathrm{C} 6$ adduct with much higher selectivity of 1:1.6:8.9 of C2:C4:C6 (see Figure 1C). Clearly, the effect of the ester substituent greatly biased the addition and served as a starting point for our study to evaluate the various effects of pyridine substituents on the regioselective preference of nucleophilic addition.

In Scheme 1A, the results of nucleophilic additions to various pyridiniums are outlined. The general reaction employs first the treatment of a parent pyridine with methyl triflate at rt, followed by the subsequent addition of 3-butenylmagnesium bromide solution at $-78^{\circ} \mathrm{C}$ (for optimization of conditions, see $\mathrm{SI}$ ). For simplicity, methyl was chosen as the $N$-alkyl substituent so that the innate reactivity of the pyridinium was observed with the least steric perturbance, but rationally, many other alkyl substituents can be employed, with similar reactivity trends (e.g. 39, vide infra). It is worth noting that the only observed electrophilic sites for addition are the 2,4 , and 6 positions of the pyridine. For monosubstituted pyridines 9-12, various substituent directing effects were observed. Esters and nitriles induced a para-directing effect (see $\mathbf{1 0}$ and 11), while the diethyl amide and bromide directed addition to the ortho positions. It is hypothesized that the amide's observed selectivity arises from chelation, while the bromide's effect is electronic in nature. The combined effects of these substituents on regioselective addition were then evaluated. First, bromides $\mathbf{1 4}$ and $\mathbf{1 5}$ showed preference for $\mathrm{C} 4$ owing to an additive directing effect of the C5 alkyl group. Dibromide 16 also showed the same contrasteric preference for $\mathrm{C} 4$ addition, albeit with reduced selectivity $(\mathrm{C} 4: \mathrm{C} 2=2.8: 1)$. For 5-bromonicotinitrile (13) and methyl 5-bromonicotinate (22), the combined directing effect resulted in preferential addition at $\mathrm{C} 6$. When other nicotinates were evaluated (18-21), the same selectivity was observed as with 22 (C6 preferred). However, the substituent at the 5 position had a drastic effect on the C4/C6 regiomeric ratio. Selectivity was enhanced with more inductively withdrawing substituents (OMe, $\mathrm{F}, \mathrm{Cl}$ ), and, similarly, alkyl substitution (Me, e.g. 18) also served to enhance preference for C6. Diester 17 also displayed reasonable selectivity for $\mathrm{C} 6$, despite lacking an ortho directing substituent (vide supra). With regard to nicotinamides, the original ortho directing effect was hypothesized to be a result of organometallic chelation. When evaluated further, 5-bromomicotninamide $\mathbf{2 3}$ showed preference for $\mathrm{C} 4$ as ester-amide $\mathbf{2 4}$ showed marginal selectivity for C6. Diamide 25 showed preference for $\mathrm{C} 2$ similar to the observed selectivity for monoamide 12.

The scope of Grignard addition was largely evaluated using pyridine 22 as a model substrate (see Scheme 1B). Notably, this substrate only gives addition at $\mathrm{C} 4$ and $\mathrm{C} 6$, but the degree of selectivity is reliant on the Grignard reagent. Initially with 3butenylmagnesium bromide, an exceptional yield (90\%) and regiomeric ratio were observed favoring C6 with 8.5 :1 selectivity giving $26 .^{41}$ A similar reactivity profile was observed when using phenethylmagnesium bromide (see 27). Surprisingly, acetal 28 was produced with a much lower C6 selectivity (2.6:1), which was hypothesized to be a result of internal chelation of the acetal oxygen to the $\mathrm{Mg}$ atom in the nucleophile. This cyclic nature of the nucleophile is thought to make it more sterically hindered than the simpler alkyl Grignard reagents used to forge 26 and 27. This steric effect was exacerbated when employing dithianylmagnesium bromide to deliver 29 with nearly equal amounts of the $\mathrm{C} 4: \mathrm{C} 6$ isomers observed. This indiscriminate selectivity was also coupled with poor yield due to the its low reactivity. Decreased yields were also observed using allyl and propargylic ${ }^{42}$ nucleophiles (30 and 31), albeit with modest selectivity for C6 in $\mathbf{3 0}$ (3.9:1). With $\mathrm{sp}^{2}$ hybridized Grignard reagents, varying regioselectivities were also observed based on size or substitution pattern. While vinylmagnesium bromide had a high C6 preference (10:1), simply incorporating a methyl group into the nucleophile decreased selectivity substantially. Utilizing 1-Propenylmagnesium bromide gave 33 with 2.2:1 C6:C4 selectivity and the addition of 2-propenylmagnsium bromide gave an even lower selectivity of $1: 1$. Addition of phenylmagnesium chloride gave a similar ratio of 1.3:1 slightly in favor of C6 (see 35). Again, larger nucleophiles, albeit $\mathrm{sp}^{2}$ hybridized, gave poorer $\mathrm{C} 6$ : $\mathrm{C} 4$ selectivity. In stark contrast, sp hybridized Grignard reagents gave high selectivities $(>20: 1)$ for C6 (36 and 37), which aligns with previously observed reactivity with acylated pyridiniums. ${ }^{32,33}$ Small modifications to the electrophilic partner were also evaluated for comparison. Combination of the tert-butyl bromonicotinate and acetal Grignard reagent delivered 38 slightly favoring $\mathrm{C} 6$ in $60 \%$ yield, with slightly diminished selectivity compared with $\mathbf{2 8}$. Activation of pyridine 22 with benzyl triflate led to adduct 39 in $67 \%$ yield and a $\mathrm{C} 6: \mathrm{C} 4$ ratio of 2.4:1. This decrease in selectivity for C6 compared to the analogous methylated product $\mathbf{2 6}$ was expected due to the increased size of the benzyl substituent. ${ }^{43}$ Finally, the Grignard reagent of ethyl 2-iodobenzoate was added in to the methylated pyridinium of methyl nicotinate (11) with exclusive regioselectivity for C6 $(>20: 1)$. This substrate was important as it had been previously studied, however, no explanation was giving for the observed regioselectivity trends. ${ }^{44}$ 
A. Pyridine Study

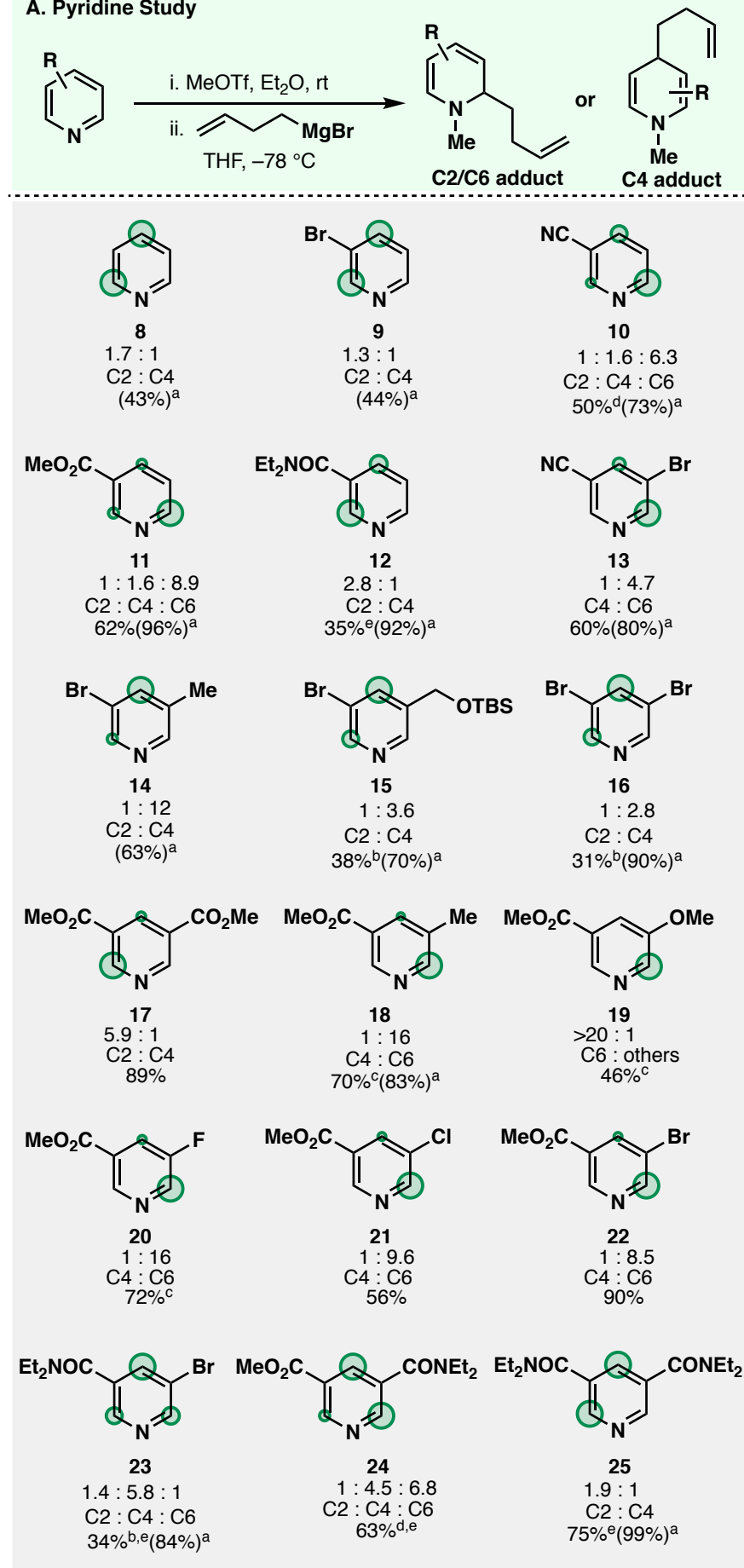

B. Grignard Study
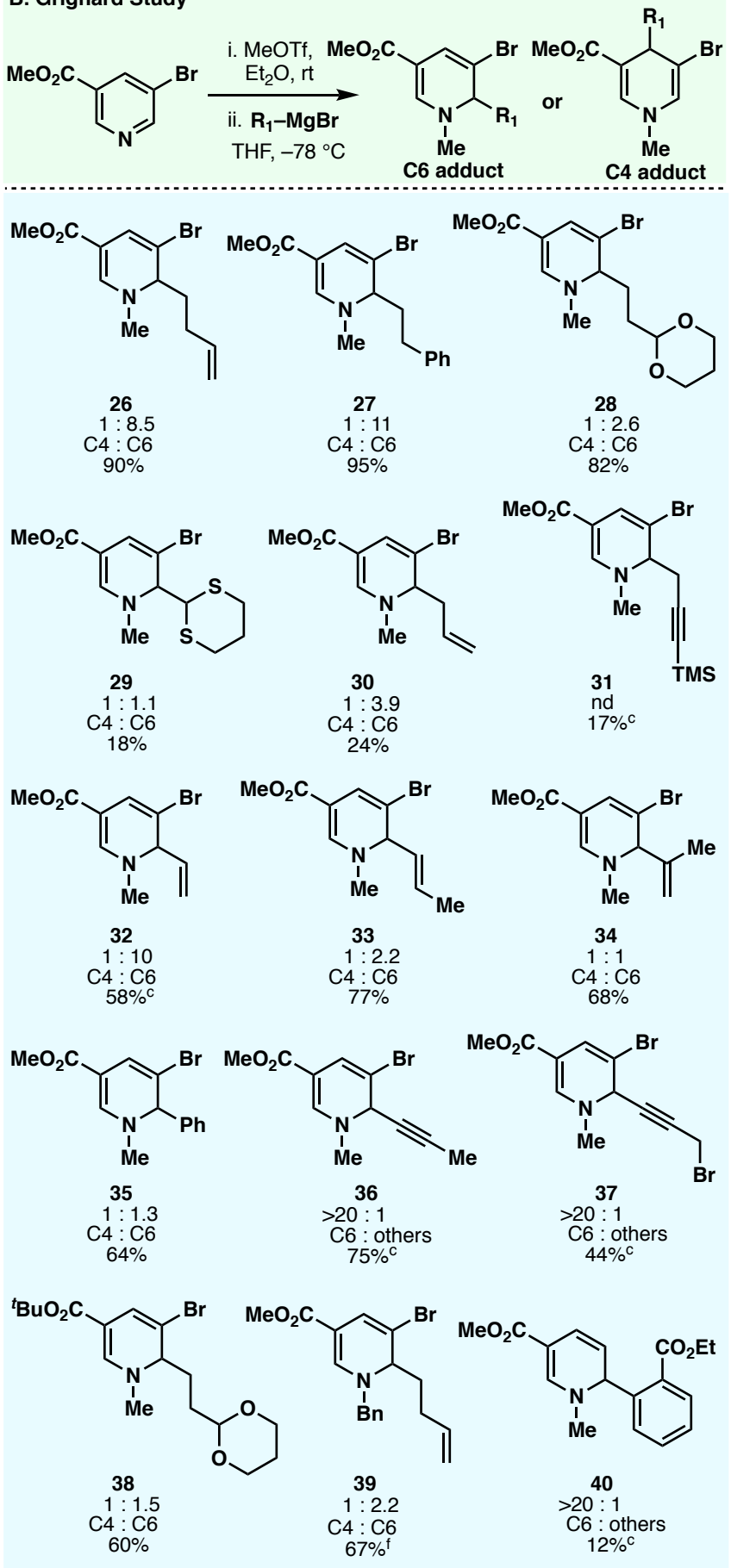

Scheme 1. (A) Regioselectivity study for addition of 3-butenylmagnesium bromide to substituted pyridiniums. Unless specified otherwise, the isolated yield is of all respective isomers ( 2 2, C4 and C6). B) Regioselectivity study for the addition of Grignard reagents to alkylpyridiniums of pyridine $\mathbf{2 2}$. Reaction conditions: MeOTf (1.0 equiv), Et $\mathrm{t}_{2} \mathrm{O}$, rt; Grignard reagent (1.0 equiv), THF, $-78{ }^{\circ} \mathrm{C}$. The regiomeric ratio was determined by ${ }^{1} \mathrm{H}$ NMR of the crude material. (a) Spectroscopic yield with trimethoxybenzene as an internal standard. (b) Isolated yield of the C4 isomer. (c) Isolated yield of C6 isomer. (d) Isolated yield of $\mathrm{C} 4$ and $\mathrm{C} 6$ isomers. (e) Methylation of substrate was performed in $\mathrm{CH}_{2} \mathrm{Cl}_{2}$. (f) BnOTf was used as an activating agent (See Supporting Information).

In order to aid practitioners in effectively harnessing pyridinium-based retrosynthetic analysis, a directing group guide and user's roadmap has been delineated in Figure 2 deduced from this study of 3 and 3,5-disubstituted pyridiniums. First, understanding the various directing group effects is paramount to employing a predictive reactivity model (Figure 2A). As unveiled in this study, chelating groups such as amides have an ortho directing effect presumably due to chelation of the organomagnesium nucleophile. ${ }^{45}$ Secondly, resonance electron-withdrawing groups like nitriles and esters primarily promote conjugate reactivity to the para position and secondarily to the ortho positions. ${ }^{28}$ Lastly, alkyl groups, halides, and heteroatoms (e.g. N,O) promote ortho addition to the pyridinium. ${ }^{46}$ It should be noted that the use of other 
organometallic species (e.g. $\mathrm{RCu}, \mathrm{R}_{2} \mathrm{Zn}$ ) are not expected to necessarily follow the above trends as this study concerns the use of Grignard reagents as nucleophiles (See Supporting Information for details).

An example of how to utilize these additive effects in a stepwise fashion is depicted in Figure 2B. Given bromonicotinamide 23, step 1 involves the identification of $\mathrm{C} 2, \mathrm{C} 4$, and C6 as innate electrophilic sites. Next, step 2 involves identifying chelating directing groups that promote proximal addition to ortho sites. Third, conjugate sites are then determined by the presence of resonance electron-withdrawing groups. Relative to 23, this preferential reactivity trend is not applicable. Lastly, the ortho directors are evaluated, which in this case, the bromine promotes addition at $\mathrm{C} 4$ and $\mathrm{C} 6$. Adding these effects together reveals a predicted selectivity for $\mathrm{C} 4$ being the highest preference with $\mathrm{C} 2$ and $\mathrm{C} 6$ being minor products of dearomative addition. Indeed, the experimental results indicate this trend in which the $N$-methylpyridinium of bromoamide 23 gives a 1.4:5.8:1 selectivity for $\mathrm{C} 2: \mathrm{C} 4: \mathrm{C} 6$. In general, these reactivity trends hold true in the present study, with regard to preferential addition. Variability is seen based on nucleophile identity, where larger nucleophiles seem to be less selective in terms of the product ratios (see 28, 29, and 38). Importantly, while selectivity can be variable in this sense, the major product observed still adheres to the guidelines depicted in Figure 2B.

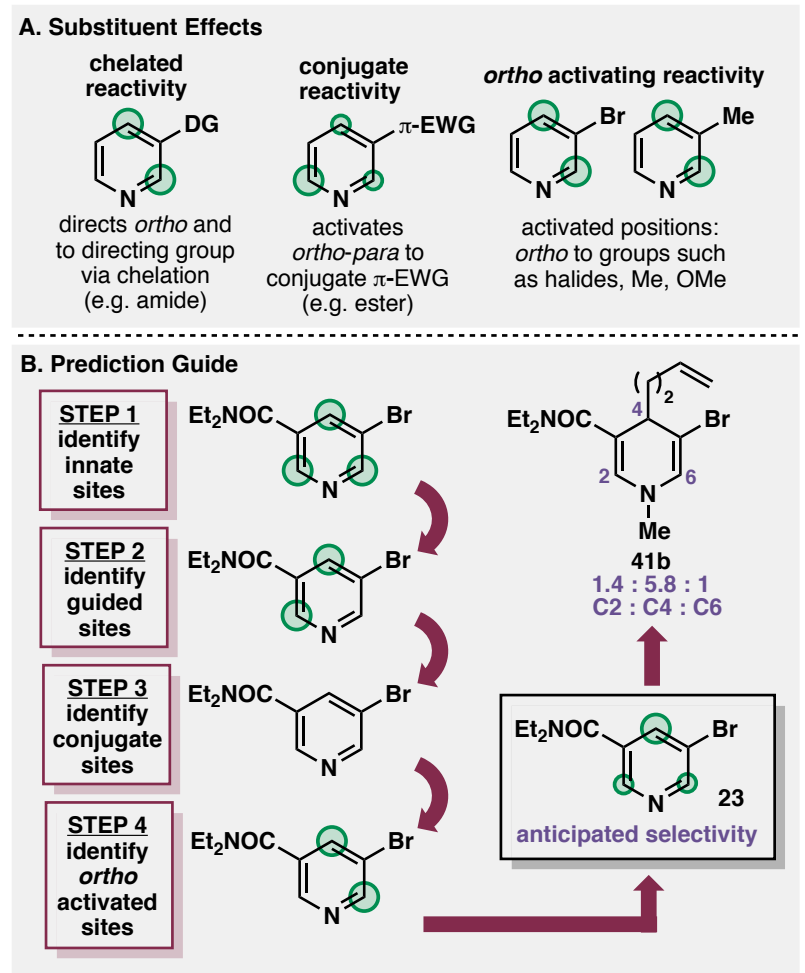

Figure 2. (A) Directing Group Effects. (B) User's guide to regioselective addition.

In order to evaluate the synthetic utility of this reactivity study, the construction of $\mathbf{3}$ was investigated. ${ }^{13}$ This compound has shown notable insecticidal activity and has shown affinity towards binding the $5 \mathrm{HT}_{7}$ serotonin receptor. First, methylation of $\mathbf{2 2}$ and addition of phenethylmagnesium bromide delivered the dearomatized addition product. This compound was then subsequently reduced with $\mathrm{LiAlH}_{4}$ to give $\mathbf{4 2}$ on gram scale in $69 \%$ yield over 2 steps. A radical annulation utilizing $\mathrm{Bu}_{3} \mathrm{SnH}$ and AIBN afforded despyrrole methyl lysergate $(43)$ in $21 \%$ yield ( $43 \%$ based on recovered starting material). This 3-step procedure compares favorably to the prior synthesis, which required 7 steps to synthesize this target. ${ }^{47-50}$ Ester saponification with aqueous $\mathrm{HCl}$ followed by an amidation protocol with CDI gave 3 in 30\% yield over 2 steps and in 5 total steps (previously 13 steps). It is worth noting that the bromide serves both as a directing group for the innate selectivity of the dearomatization and as a handle for $\mathrm{C}-\mathrm{H}$ annulation. The selectivity of the hydride reduction step is also remarkable, giving dihydropyridine $\mathbf{4 2}$, in that very little ester reduction is observed and the reduction regiochemistry is absolute.

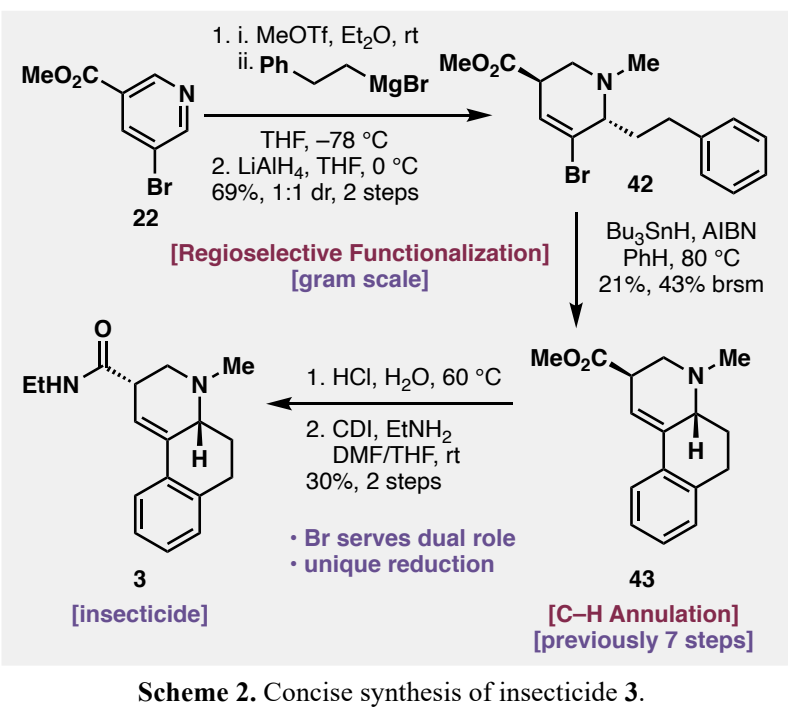

In conclusion, this study has shown the innate reactivity trends associated with $N$-alkyl pyridinium electrophiles. The effects of substitution patterns have revealed outcomes in organometallic additions that are systematic and easily employed in first-pass retrosynthetic analyses. These data serve to delineate reactivity that, prior to this study, were limited and not conclusively understood. In some instances of the current work, the selectivity proved quite high, and could be easily utilized in multistep synthesis, as shown by the concise construction of 3. It is hoped that the lessons learned will be broadly helpful to practitioners ambitious to utilize the inherent advantages of dearomative synthetic approaches. The expansion upon these findings and their application to future synthetic targets is anticipated to be reported by our group in due course.

\section{ASSOCIATED CONTENT}

\section{Supporting Information}

The Supporting Information is available free of charge on the ACS Publications website.

Experimental Procedures and analytical data $(1 \mathrm{H}, 13 \mathrm{C}$ NMR, MS) for all new compounds (PDF)

Crystallographic data (CIF)

\section{AUTHOR INFORMATION}




\section{Corresponding Author}

Joel M. Smith - Department of Chemistry and Biochemistry, Florida State University, Tallahassee, FL 32306, United States; ORCID: 0000-0002-1108-4751; Email: smith@chem.fsu.edu.

\section{Authors}

Brian J. Knight - Department of Chemistry and Biochemistry, Florida State University, Tallahassee, FL 32306, United States Zachary A. Tolchin - Department of Chemistry and Biochemistry, Florida State University, Tallahassee, FL 32306, United States

\section{Notes}

The authors declare no competing financial interest.

\section{ACKNOWLEDGMENT}

Financial support provided by Florida State University. We thank Xinsong Lin for assistance with X-ray data collection and structure determination. We thank Prof. James Frederich and Prof. Igor Alabugin for vibrant and helpful discussions.

\section{REFERENCES}

(1) Corey, E. J. Molecules and Medicine; John Wiley \& Sons: Hoboken, 2007.

(2) Nicolaou, K. C.; Montagnon T. Molecules that Changed the World; Wiley-VCH: Weinheim, 2008.

(3) Heterocycles in Natural Product Synthesis; Majumdar, K. C.; Chattopadhyay, S. K., Eds.; Wiley-VCH: Weinheim, 2011.

(4) Modern Heterocyclic Chemistry; Alvarez-Builla, J.; Vaquero, J. J.; Barluenga, J., Eds.; Wiley-VCH: Weinheim, 2011.

(5) Joule, J. A.; Mills, K. Heterocyclic Chemistry at a Glance; John Wiley \& Sons: West Sussex, 2013, Vol. 4.

(6) Vitaku, E.; Smith, D. T.; Njardarson, J. T. Analysis of the Structural Diversity, Substitution Patterns, and Frequency of Nitrogen Heterocycles among U.S. FDA Approved Pharmaceuticals. J. Med. Chem. 2014, 57, 10257-10274.

(7) Huffman, B. J.; Shenvi, R. A. Natural Products in the "Marketplace": Interfacing Synthesis and Biology. J. Am. Chem. Soc. 2019, 141, 3332-3346.

(8) Baumann, M.; Baxendale, I. R. An overview of the synthetic routes to the best selling drugs containing 6-membered heterocycles. Beilstein J. Org. Chem. 2013, 9, 2265-2319.

(9) Vardanyan, R. Piperidine-Based Drug Discovery; Elsevier: Amsterdam, 2017.

(10) Liu, G.-Q.; Opatz, T. Recent Advances in the Synthesis of Piperidines: Functionalization of Preexisting Ring Systems. In Advances in Heterocyclic Chemistry; Scriven, E. F. V.; Ramsden, C. A., Eds.; Academic Press: San Diego, 2018, Vol. 125, pp 107234.

(11) Ashok, P.; Lathiya, H.; Murugesan, S. Manzamine alkaloids as antileishmanial agents: A review. Eur. J. Med. Chem. 2014, 97, 928-936.

(12) Devereaux, A. L.; Mercer, S. L.; Cunningham, C. W. DARK Classics in Chemical Neuroscience: Morphine. ACS Chem. Neurosci. 2018, 9, 2395-2407.

(13) Rowley, E. G.; Sehgel, S.; Crawford, S. D. Insecticidal Despyrrole Analogs of Lisuride and Lysergamides. US Patent WO 2007/128409 A1, November 15, 2007.

(14) Germann, D.; Ma, G.; Han, F.; Tikhomirova, A. Chapter Eight - Paroxetine Hydrochloride. In Profiles of Drug Substances, Excipients, and Related Methodology; Brittain H. G., Ed.; Academic Press: San Diego, 2013, Vol. 38, pp 367-406.

(15) Bull, J. A.; Mousseau, J. J.; Pelletier, G.; Charette. A. B. Synthesis of Pyridine and Dihydropyridine Derivatives by Regioand Stereoselective Addition to $\mathrm{N}$-Activated Pyridines. Chem. Rev. 2012, 112, 2642-2713.
(16) Shenvi, R. A.; O’Malley, D. P.; Baran. P. S. Chemoselectivity: The Mother of Invention in Total Synthesis. Acc. Chem. Res. 2009, 42, 530-541.

(17) Nebe, M. M.; Opatz. T. Chapter Five - Synthesis of Piperidines and Dehydropiperidines: Construction of the Six-Membered Ring. In Advances in Heterocyclic Chemistry; Scriven, E. F. V.; Ramsden C. A., Eds.; Academic Press: San Diego, 2017, Vol. 122, pp 191-244.

(18) Bari, A.; Iqbal, A.; Khan, Z. A.; Shahzad, S. A.; Yar. M. Synthetic approaches toward piperidine related structures: A review. Syn. Commun. 2020, 1-18.

(19) Müller, T. E.; Hultzsch, K. C.; Yus, M.; Foubelo, F.; Tada, M. Hydroamination: Direct Addition of Amines to Alkenes and Alkynes. Chem. Rev. 2008, 108, 3795-3892.

(20) Gaich. T.; Baran. P. S. Aiming for the Ideal Synthesis. J. Org. Chem. 2010, 75, 4657-4673.

(21) Crossley, S. W. M.; Shenvi. R. A. A Longitudinal Study of Alkaloid Synthesis Reveals Functional Group Interconversions as Bad Actors. Chem. Rev. 2015, 115, 9465-9531.

(22) Roche, S. P.; Porco, J. A. Dearomatization Strategies in the Synthesis of Complex Natural Products. Angew. Chemie, Int. Ed. 2011, 50, 4068-4093.

(23) Ding, Q.; Zhou, X.; Fan, R. Recent advances in dearomatization of heteroaromatic compounds. Org. Biomol. Chem. 2014 $12,4807-4815$.

(24) Bertuzzi, G.; Bernardi, L.; Fochi, M. Nucleophilic Dearomatization of Activated Pyridines. Catalysts 2018, 8, 1-36.

(25) Wong, D.; Wang, Z.; Liu, Z.; Huang, M.; Hu, J.; Yu. P. Strategic $\mathrm{C}-\mathrm{C}$ Bond-Forming Dearomatization of Pyridines and Quinolines. Org. Lett. 2019, 21, 4459-4463.

(26) Robinson, D. J.; Spurlin, S. P.; Gorden, J. D.; Karimov, R. R. Enantioselective Synthesis of Dihydropyridines Containing Quaternary Stereocenters Through Dearomatization of Pyridinium Salts. ACS Catal. 2020, 10, 51-55.

(27) Sowmiah, S.; Esperança, J. M. S. S.; Rebelo, L. P. N.; Afonso, C. A. M. Pyridinium salts: from synthesis to reactivity and applications. Org. Chem. Front. 2018, 5, 453-493.

(28) To our knowledge, a singular study investigated the regioselectivity of Grignard additions into 3,5-disubstituted alkylpyridiniums, see, Lyle, R. E.; White, E. Reaction of organometallic reagents with pyridinium ions. J. Org. Chem. 1971, 36, 772-777. (29) Comins, D. L.; Brown, J. D. Addition of metallo enolates to 1 -acylpyridinium salts. A short synthesis of $( \pm)$-epi-lupinine. Tetrahedron Lett. 1986, 27, 2219-2222.

(30) Comins, D. L.; Brown, J. D. Addition of Grignard reagents to 1-acyl-4-methoxypyridinum salts. An approach to the synthesis of quinolizidinones. Tetrahedron Lett. 1986, 27, 4549-4552.

(31) Comins, D. L.; Myoung, Y. C. Synthesis and synthetic utility of 1-acyl-5-(trialkylsilyl)-1,2-dihydropyridines. A synthesis of (士)-elaeokanine A. J. Org. Chem. 1990, 55, 292-298.

(32) Yamaguchi, R.; Nakazono, Y.; Kawanisi, M. On the regioselectivity of the reaction of $N$-methoxycarbonylpyridinium chloride with Grignard reagents: highly regioselective synthesis of 2substituted $N$-methoxycarbonyl-1,2-dihydropyridines. Tetrahedron Lett. 1983, 24, 1801-1804.

(33) Yamaguchi, R.; Hata, E.; Matsuki, T.; Kawanisi, M. An efficient regio- and stereoselective synthesis of $( \pm)$-monomorine I via the highly regioselective $\alpha$-alkynylation of a 1-acylpyridinium salt. J. Org. Chem. 1987, 52, 2094-2096.

(34) Donohoe, T. J.; Connolly, M. J.; Walton, L. Regioselective Nucleophilic Addition to Pyridinium Salts: A New Route to Substituted Dihydropyridones. Org. Lett. 2009, 11, 5562-5565.

(35) Charette, A. B.; Grenon, M.; Lemire, A.; Pourashraf, M.; Martel, J. Practical and Highly Regio- and Stereoselective Synthesis of 2-Substituted Dihydropyridines and Piperidines: Application to the Synthesis of (-)-Coniine. J. Am. Chem. Soc. 2001, $123,11829-11830$.

(36) Lemire, A.; Beaudoin, D.; Grenon, M.; Charette, A. B. [4+2] Cycloaddition of 2-Substituted 1,2-Dihydropyridines with 
Nitrosobenzene: Asymmetric Synthesis of trans-2-Substituted 3Amino-1,2,3,6-tetrahydropyridines. J. Org. Chem. 2005, 70, 2368-2371.

(37) Charette, A. B.; Mathieu, S.; Martel, J. Electrophilic Activation of Lactams with $\mathrm{Tf}_{2} \mathrm{O}$ and Pyridine: Expedient Synthesis of ( \pm )-Tetraponerine T4. Org. Lett. 2005, 7, 5401-5404.

(38) Comins, D. L.; Goering, R. R; Joseph, S. P.; O'Connor, S. Asymmetric synthesis of 2-alkyl(aryl)-2,3-dihydro-4-pyridones by addition of Grignard reagents to chiral 1-acyl-4-methoxypyridinium salts. J. Org. Chem. 1990, 55, 2574-2576.

(39) Comins, D. L.; Joseph, S. P.; Goehring, R. R. Asymmetric synthesis of 2-alkyl (aryl)-2, 3-dihydro-4-pyridones by addition of Grignard reagents to chiral 1-acyl-4-methoxypyridinium salts. J. Am. Chem. Soc. 1994, 116, 4719-4728.

(40) Streith, J.; Boiron, A.; Sifferlen, T.; Strehler, C.; Tschamber, T. A simple asymmetric synthesis of 2-substituted 2,3-dihydro-4pyridones. Tetrahedron Lett. 1994, 35, 3927-3930.

(41) $\mathrm{Cu}$ and $\mathrm{Zn}$ organometallic species were investigated. See SI for details.

(42) Dihydropyridine 31 was isolated as a 9:1 mixture of the propargyl and allenyl addition products, respectively.

(43) Holm, T. Mechanism of the Grignard Addition Reaction. XV. The Reaction of Grignard Reagents with Benzylpyridinium Chloride. Acta Chemica Scandinavica. 1991, 45, 276-279.

(44) Bennasar, M.-L.; Roca, T.; Monerris, M.; Juan, C.; Bosch, J. Synthesis of 4-functionalized aryl-3,5-diacyl-1,4-dihydropyridines. Tetrahedron 2002, 58, 8099-8106.
(45) Schultz, A. G.; Flood, L. Regio- and stereoselective control in the addition of Grignard reagents to the pyridine ring system. J. Org. Chem. 1986, 51, 838-841.

(46) Lemire, A.; Grenon, M.; Pourashraf, M.; Charette, A. B. Nucleophilic Addition to 3-Substituted Pyridinium Salts: Expedient Syntheses of (-)-L-733,061 and (-)-CP-99,994. Org. Lett. 2004, 6, 3517-3520.

(47) Kiguchi, T.; Hashimoto, C.; Naito, T.; Ninomiya, I. A Route for Total Synthesis of Ergot Alkaloids. Synthesis of the Despyrrole Analogs of Methyl Lysergate, Isolysergol, and Isofumigaclavine A. Heterocycles 1982, 19, 1873-1877.

(48) Ninomiya, I.; Hashimoto, C.; Kiguchi, T.; Naito, T. Photocyclisation of enamides. Part 22. Syntheses of the despyrrole analogues of some ergot alkaloids including methyl lysergate and isofumigaclavine A. J. Chem. Soc. Perkin Trans. I 1984, 29112917.

(49) Ninomiya, I.; Hashimoto, C.; Kiguchi, T.; Naito, T. Photocyclisation of enamides. Part 24. Total synthesis of ( \pm )-isofumigaclavine B and ( \pm )-lysergic acid. J. Chem. Soc. Perkin Trans. I 1985, 941-948.

(50) Ninomiya, I.; Hashimoto, C.; Kiguchi, T.; Naito. T. Photocyclization of Enamides. XXV. : Syntheses of Despyrrole Analogues of Ergot Alkaloids Including Elymoclavine, Lysergol, Isolysergol, Lysergine, Isolysergine, Fumigaclavine, Agroclavine, and Lysergene. Chem. Pharm. Bull. 1986, 34, 27992809. 
TOC Graphic

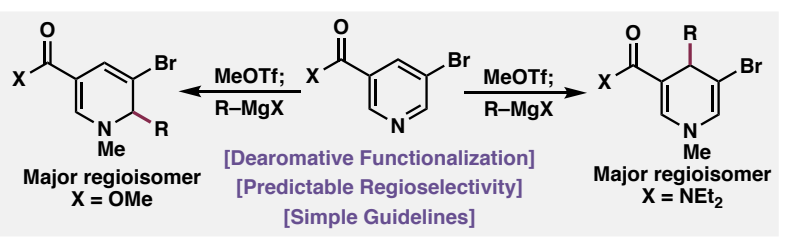

